\section{Ausgezeichnet: Beste Kasuistik 2016}

Zum ersten Mal wurde in diesem Jahr der Kasuistikpreis für die beste Kasuistik aus den vergangenen zwei Jahren vergeben. Ausgezeichnet wurde der in der September-Ausgabe 2016 veröffentlichte Fallbericht von Dr. Frederik Frenzen et al. mit dem Titel „Pulmonale Pichia guilliermondii-Infektion - Eine seltene Differenzialdiagnose pulmonaler Rundherde“" (Pneumologie 2016; 70: 605-607).

Bei der Wahl des Preisträgers war sich die Jury, bestehend aus den Herausgebern der Zeitschrift, Prof. Tom Schaberg und Prof. Santiago Ewig, schnell einig: „Der Fallbericht stellt eine sorgfältige Abklärung von pulmonalen Rundherden dar, bei der ein mykotischer Erreger (Pichia guilliermondi) detektiert wurde, der in dieser Form in Europa bisher noch nicht beschrieben war. Der Fallbericht ist kon- zise und informativ verfasst und erweitert mit dem Erregernachweis die differenzialdiagnostischen Möglichkeiten bei der Abklärung infektiologisch bedingter pulmonaler Rundherde.“

Die Freude über die mit einem Preisgeld von $500 €$ und einem Jahresabonnement der Zeitschrift verbundene Auszeichnung war bei Dr. Frenzen groß. Frau Dr. Röder, Kollegin und Koautorin der Kasuistik aus Halle (Saale), brachte den Facharzt für Innere Medizin und Notfallmediziner auf die Idee, den „kniffeligen Fall aus der Notaufnahme, der noch in keinem Schulbuch steht", als Publikation bei der Pneumologie einzureichen. Dr. Frenzen (Jahrgang 1980) studierte an der MartinLuther-Universität zu Halle-Wittenberg Humanmedizin. Als Assistenzarzt verbrachte er ein halbes Jahr an der Klinik für Innere Medizin und Infektiologie am Christian Medical College Hospital in Vellore in Indien. Seit 2014 ist er Facharzt für Innere Medizin an der Medizinischen Klinik und Poliklinik am Universitätsklini- kum in Dresden, wo er sowohl auf der Intensivstation als auch auf der pneumologischen Normalstation sowie im Bereich der Bronchoskopie und pneumologischen Funktionsdiagnostik tätig ist.

Auch im kommenden Jahr wird es wieder eine Verleihung des Kasuistikpreises geben. Bewerbungen können noch bis Ende des Jahres per E-Mail eingereicht werden bei Prof. Tom Schaberg unter Schaberg@diako-online.de.

Daniela Sandrock, Stuttgart 